\title{
Possible H-Mode Transition with Vertically Placed Limiters
}

\author{
by \\ Tihiro Ohkawa \\ GA Technologies Inc. San Diego, California USA 92138
}

(Received February 25, 1987)

\begin{abstract}
The possibility of producing the $\mathrm{H}$-mode in the limiter discharge of tokamaks is discussed. Except for the large magnetic shear of divertor configurations, the limiters placed vertically appear to mimic the diverted configuration.
\end{abstract}

It has been observed that the threshold heating power for the transition to the $\mathrm{H}$ mode depends on the position of the divertor null point for a given direction of a toroidal magnetic field. The phenomenon has been explained by F. Hinton ${ }^{(1)}$ as the neoclassical effect on the ion heat transport. The Pfirsch-Schlüter parallel ion heat flow adds to or subtracts from the parallel heat drain depending on the position of the heat sink. The divertor configurations with double nulls or with a single null on the median plane do not take advantage of this effect and thus have a higher threshold heating power. The configuration with a single null on the median plane outboard side not only suffers from the absence of this effect but also stays in the collisional regime even at high ion temperature because of very small bounce frequency. (2)

Tokamak plasmas with limiters usually employ the limiters at outboard or inboard side locations. The heat sink is essentially symmetric with respect to the median plane and the effects of Pfirsch-Schlüter heat flow are absent. The plasma with the inboard limiter is similar to the plasma with the single null divertor placed at the inboard side, provided that the limiter is extremely clean and recycling is minimal. Once the edge ion temperature is increased with high power heating, the trapped ions can survive in the edge region because their orbits do not intersect the limiter. The plasma with the outboard limiter is similar to the plasma with the single null divertor placed at the outboard side. The limiter eliminates the trapped ions near the scrape-off layer.

An interesting question is what if the limiter is placed at the top or the bottom of the plasma. The neoclassical effects are present and a reasonable heating power should be sufficient to exceed the threshold power. Will the plasma make the transition to the H-mode?

The major difference is that the divertor configuration has a large magnetic shear near the separatrix. If the large ion temperature gradient at the edge can be sustained stably only with the large magnetic shear, the limiter discharges can not be in the H-mode. The fact that there has not been a clear demonstration of the H-mode in JT-60 and that the divertor discharges can be in the L-mode shows that the presence of the separatrix is not a sufficient condition for the H-mode. Experiments are needed to find out if it is a necessary condition. 
The experimental observations showed that the null point or the part of the separatrix bounding plasma must have a certain distance from the wall to obtain the H-mode. If it is related to the recycling at the wall, the limiter tip inserted some distance from the wall should be effective. If it means that any material must be some distance from the plasma, the limiter will not produce the $\mathrm{H}$-mode.

The particle drain asymmetric with respect to the median plane affects the transport of impurity ions. ${ }^{(3)}$ The Pfirsch-Schlüter flow of hydrogen ions brings the impurity ions through collisions to the side where the drift due to the gradient of the magnetic field strength is inward. When the hydrogen ion drainage flow is counter to the Pfirsch-Schlüter flow, the inward transport of the impurity ions is reduced. The configurations having a lower threshold heating power thus have a lower impurity inflow rate.

Since it is very costly and time-consuming to modify the magnetic configurations of large devices, the $\mathrm{H}$-mode discharges with limiters are of interest. The discharges with the vertical limiters have many ingredients in common with the diverted $\mathrm{H}$-mode discharges. They are the presence of the neoclassical effects lowering the heating power threshold, the survivability of the hot trapped ions near the edge, the distance between the plasma edge to the wall and the reduced impurity inflow. Experimental tests in medium-sized devices with the capability of divertor configuration may tell us whether the magnetic shear near the separatrix is a necessary condition or not. A discharge with a vertical limiter can be compared to a diverted discharge in the same device. The proposed experiment not only explores the possibility of the $\mathrm{H}$-mode with the limiter but also helps to understand the $\mathrm{H}$-mode.

\section{REFERENCES}

1) F. L. Hinton, Nucl. Fusion 25, 1457 (1985).

2) T. Ohkawa and F. L. Hinton, "Effect of $x$-Point Location on Edge Confinement in Diverted Tokamaks," GA Technologies Report GA-A18644, November 1986.

3) T. Ohkawa, Kakuyugo-Kenkyu 32, 67 (1974). 